Max-Planck-Institut für demografische Forschung

Max Planck Institute for Demographic Research

Konrad-Zuse-Strasse 1 - D-18057 Rostock - GERMANY

Tel +49 (0) 3812081 - 0; Fax +49 (0) 3812081 - 202;

http://www.demogr.mpg.de

MPIDR TECHNICAL REPORT 2016-001

APRIL 2016

\title{
R Programs for Writing HMD \\ Life Tables and HMD Death Rates \\ to Pooled Data Files
}

Vladimir M. Shkolnikov (shkolnikov@demogr.mpg.de)

Dmitri A. Jdanov (jdanov@demogr.mpg.de)

(C) Copyright is held by the authors.

Technical reports of the Max Planck Institute for Demographic Research receive only limited review. Views or opinions expressed in technical reports are attributable to the authors and do not necessarily reflect those of the Institute. 


\title{
R Programs for Writing HMD Life Tables or HMD Death Rates to Pooled Data File
}

Vladimir M. Shkolnikov (shkolnikov@demogr.mpg.de)

Dmitri A. Jdanov (jdanov@demogr.mpg.de)

\begin{abstract}
The Human Mortality Database (HMD) website provides an opportunity for downloading output data as zipped files. Unzipping these files returns folders that contain numerous data files, which correspond to age-by-year data formats $(1 \mathrm{x} 1,5 \mathrm{x} 1,1 \mathrm{x} 5,5 \mathrm{x} 5, \ldots)$, sexes, and dozens of country-populations. For many analyses, however, it would be more practical to operate with long data file containing many populations rather than large number of population-specific files. Herewith, we provide two simple programs for: 1) pooling together life tables' for many countries and years in a single file and 2) death rates for many countries and years in a single file.
\end{abstract}




\section{Motivation}

The world leading resource on mortality data the Human Mortality Database (HMD) provides detailed data series for more than 40 population and subpopulation. Despite on high quality of output data and easy-to-use interface the range of output statistics does not include files aggregated over countries. But the website provides an opportunity for downloading output data as zipped files by indicators. Unzipping these files returns folders that contain numerous data files, which correspond to age-by-year data formats $(1 \times 1,5 \times 1,1 \times 5,5 \times 5, \ldots)$, sexes, and dozens of country-populations. In this technical report we present two R scripts for: 1) pooling together life tables for many countries and years in a single file and 2) pooling together death rates for many countries and years in a single file. Using these scripts is extremely simple and practically does not require any knowledge of R. Scripts were tested under R version 3.1.

The aggregation of HMD files requires two steps: 1) downloading data from the HMD and 2) running the respective R script. Below we describe both steps.

\section{Preparation of input data: downloading zipped files from the HMD website}

In addition to possibility of downloading single data files for chosen mortality indicator, sex, ageby-year format and country, the HMD provides possibility to download zip archives containing many data files. The page with zip files by all HMD output indicators is available at http://www.mortality.org/cgi-bin/hmd/hmd_download.php . There is also link to this page (“Zipped Data Files") in the "HMD Main Menu" on left-hand-side of the HMD face webpage. Clicking on this item brings user to the list of downloadable zipped data files (see Figure 1). Among these files, we will be interested in period and cohort Life Tables and in period and cohort Death Rates. 


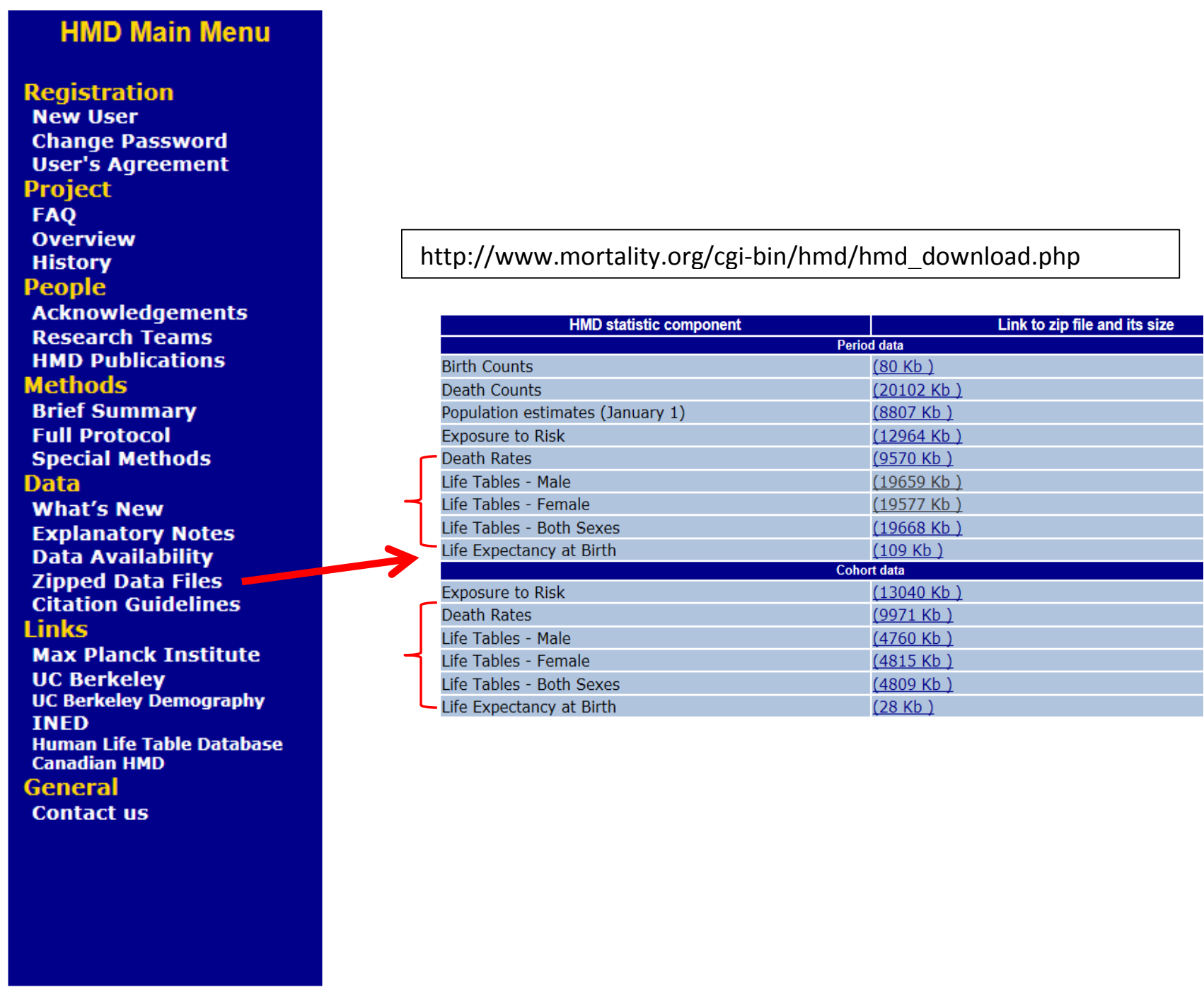

Figure 1. Getting to downloadable zipped files with period and cohort life tables and with period and cohort death rates from the HMD face page

Right clicking on links in the right column of the table (Figure 1) enables to download corresponding zipped files of death rates or life tables. To finish the process of data preparation it is enough just unzip the file in a new empty folder.

\section{R scripts}

\section{Installation}

The technical report includes the zip file with two R scripts. Input and output data corresponding to the two examples given below can be provided upon request. There is no need to install any packages before running these scripts. It is enough just to unzip the zip file and source both R files (Pool_HMD_LT.r and Pool_HMD_Mx.r) either using menu or by typing two following command in R console: 
source(“/path-to-the-script/Pool_HMD_LT.r”)

source(“/path-to-the-script/Pool_HMD_Mx.r”)

where /path-to-the-script/ is the full path to the R scripts (in case they are not in the R working directory).

\section{Usage}

To aggregate life table files:

Pool.HMD.LT.files (DataDir = '.', OutputDir = './pooled', OutputFile = “agg.csv”, Separator = ",")

To aggregate death rates

Pool.HMD.LT.files (DataDir = '.', OutputDir = './pooled', OutputFile = “agg.csv”, Separator = ",") Arguments:

DataDir - Folder with HMD files to be aggregated. Please check that it does not contain any other (non-HMD) files

OutputDir - Folder, where the output file will be saved

OutputFile - File name of the output file with aggregated data

Separator - Separator of data fields in the output file

\section{Examples}

Herewith, we illustrate use of the R scripts Pool.HMD.Mx.files and Pool.HMD.LT.files by two examples.

\section{Example 1. Pooling country-specific death rates}

Right-click on the link corresponding to "Death Rates" in the upper (period) part of the table on the webpage http://www.mortality.org/cgi-bin/hmd/hmd_download.php and download the file death_rates.zip. Unzipping of this file produces five folders $M x \_1 \times 1, M x \_1 \times 5, M x \_1 \times 10, M x \_5 \times 1$, $M x \_5 \times 5, M x \_5 x 10$. Each of these folders contains country-files of death rates in respective age-byyear formats. For example, the folder $M x \_1 x 1$ contains 47 country-files ${ }^{1}$ of single-age and singlecalendar-year death rates named:

AUS.Mx_1x1.txt, AUT.Mx_1x1.txt, BEL.Mx_1x1.txt, .., USA.Mx_1x1.txt.

To build the pooled file, which will contain single-age- and single-year-specific death rates for all country-populations, it is necessary to copy all the files (AUS.Mx_1x1.txt, AUT.Mx_1x1.txt, BEL.Mx_1x1.txt, ..., USA.Mx_1x1.txt ) into certain “input” folder (say) c:VnputData1 \, open the RStudio or simple R-console and after sourcing of the file Pool.HMD.Mx.files.R (see section Instalation for details) call the function

\footnotetext{
${ }^{1}$ The actual number of data series as of April 2016. Might be more in future.
} 
Pool.HMD.Mx.files("c:/InputData1", "c:/OutputData1", "Death-rates1x1.csv", Separator=",").

During execution of the code, the following lines will appear in the Console window:

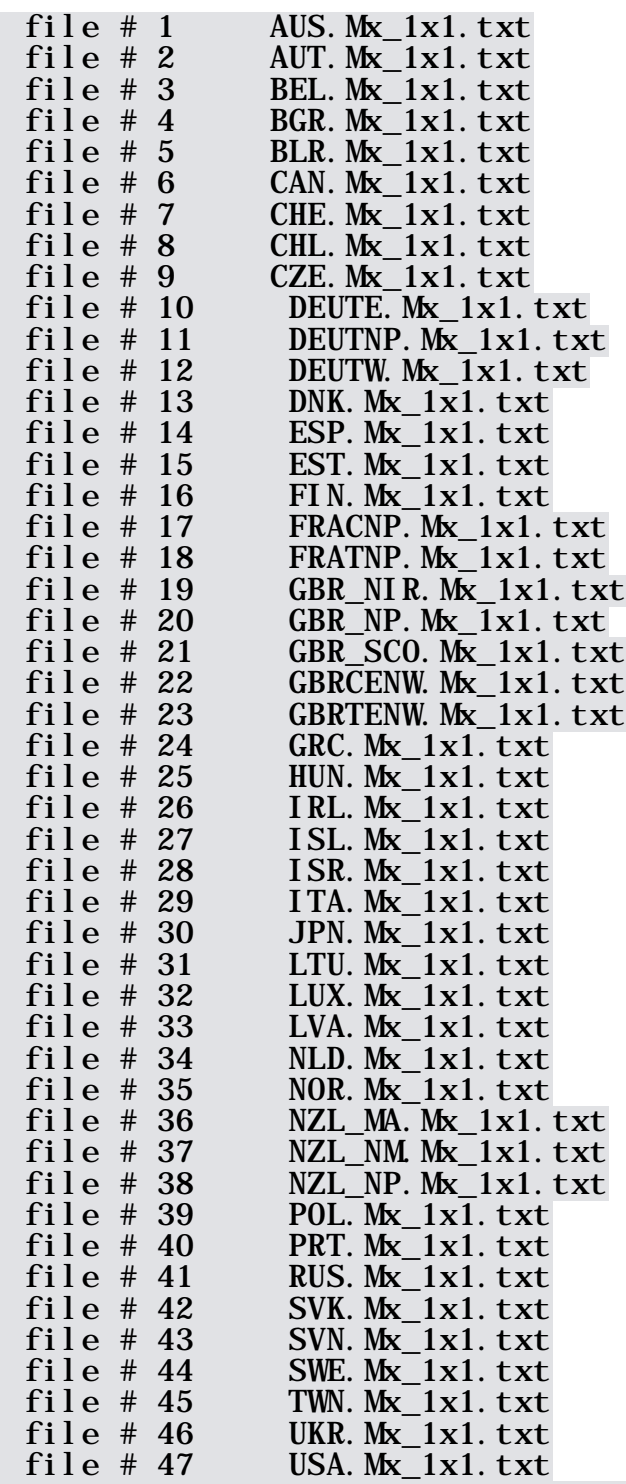

The output file Death-rates1x1.csv appears in the output folder c:\OutputData1. The file contains death rates for all country-populations:

$\begin{array}{lllcrccc}\text { DType } & \text { Scale } & \text { Popul } & \text { Year } & \text { Age } & \text { Female } & \text { Male } & \text { Total } \\ \text { Mx } & 1 \times 1 & \text { AUS } & 1921 & 0 & 0.059987 & 0.076533 & 0.068444 \\ \text { Mx } & 1 \times 1 & \text { AUS } & 1921 & 1 & 0.012064 & 0.014339 & 0.013225 \\ \text { Mx } & 1 \times 1 & \text { AUS } & 1921 & 2 & 0.005779 & 0.006047 & 0.005916 \\ \text { Mx } & 1 \times 1 & \text { AUS } & 1921 & 3 & 0.002889 & 0.004197 & 0.003554 \\ \text { Mx } & 1 \times 1 & \text { AUS } & 1921 & 4 & 0.003254 & 0.003254 & 0.003254 \\ \text { Mx } & 1 \times 1 & \text { AUS } & 1921 & 5 & 0.002517 & 0.002509 & 0.002513 \\ \text { Mx } & 1 \times 1 & \text { AUS } & 1921 & 6 & 0.002485 & 0.002218 & 0.00235 \\ M x & 1 \times 1 & \text { AUS } & 1921 & 7 & 0.001812 & 0.001924 & 0.001869 \\ M x & 1 \times 1 & \text { AUS } & 1921 & 8 & 0.001384 & 0.001914 & 0.001651\end{array}$




\begin{tabular}{|c|c|c|c|c|c|c|c|}
\hline Mx & $1 \times 1$ & AUS & 1921 & 9 & ๑. 001371 & ๑. 001419 & $\odot .001395$ \\
\hline$M x$ & $1 \times 1$ & AUS & 1921 & 10 & $\odot .001249$ & $\odot .001508$ & 0.00138 \\
\hline Mx & $1 \times 1$ & AUS & 1921 & 11 & $\odot .001159$ & $\odot . \odot \odot 21 \odot 4$ & $\odot .001639$ \\
\hline Mx & $1 \times 1$ & AUS & 1921 & 12 & $\odot .001128$ & $\odot .001639$ & $\odot .0 \odot 1387$ \\
\hline Mx & $1 \times 1$ & AUS & 1921 & 13 & $\odot .001417$ & $\odot .001547$ & 0.001482 \\
\hline Mx & $1 \times 1$ & AUS & 1921 & 14 & $\odot .001435$ & $\odot .001809$ & $\odot .001625$ \\
\hline Mx & $1 \times 1$ & AUS & 1921 & 15 & $\odot .001602$ & $\odot .001463$ & $\odot .001531$ \\
\hline Mx & $1 \times 1$ & AUS & 1921 & 16 & $\odot .00181$ & $\odot . \odot \odot 2 \odot 22$ & ๑.001918 \\
\hline Mx & $1 \times 1$ & AUS & 1921 & 17 & $\odot .0 \odot 2166$ & $\odot . \odot \odot 2518$ & $\odot .0 \odot 2343$ \\
\hline Mx & $1 \times 1$ & AUS & 1921 & 18 & $\odot . \odot \odot 2477$ & $\odot . \odot \odot 2569$ & 0.002523 \\
\hline Mx & $1 \times 1$ & AUS & 1921 & 19 & $\odot .0 \odot 2201$ & $\odot . \odot \odot 2415$ & $\odot .0 \odot 23 \odot 8$ \\
\hline Mx & $1 \times 1$ & AUS & 1921 & 20 & $\odot . \odot \odot 2976$ & $\odot .0 \odot 2496$ & $\odot .0 \odot 2737$ \\
\hline Mx & $1 \times 1$ & AUS & 1921 & 21 & $\odot . \odot \odot 2542$ & $\odot .003257$ & $\odot .0 \odot 2896$ \\
\hline Mx & $1 \times 1$ & AUS & 1921 & 22 & $\odot .0 \odot 2535$ & $\odot .003347$ & $\odot .002934$ \\
\hline Mx & $1 \times 1$ & AUS & 1921 & 23 & $\odot .003258$ & $\odot .003237$ & $\odot . \odot \odot 3248$ \\
\hline$M x$ & $1 \times 1$ & AUS & 1921 & 24 & $\odot .0 \odot 3139$ & $\odot .0 \odot 3657$ & 0.003388 \\
\hline
\end{tabular}

\begin{tabular}{|c|c|c|c|c|c|c|c|}
\hline Mx & $1 \times 1$ & AUS & 1921 & 103 & $\odot$ & $\odot .4 \odot 4313$ & 0.212164 \\
\hline $\mathrm{Mx}$ & $1 \times 1$ & AUS & 1921 & 104 & 1.039861 & 0.436999 & 0.615385 \\
\hline$M x$ & $1 \times 1$ & AUS & 1921 & 105 & . & $\odot$ & $\odot$ \\
\hline$M x$ & $1 \times 1$ & AUS & 1921 & 106 & . & $\odot$ & $\odot$ \\
\hline$M x$ & $1 \times 1$ & AUS & 1921 & 107 & . & $\odot$ & $\odot$ \\
\hline$M x$ & $1 \times 1$ & AUS & 1921 & 108 & . & $\odot$ & $\odot$ \\
\hline$M x$ & $1 \times 1$ & AUS & 1921 & 109 & . & $\odot$ & $\odot$ \\
\hline$M x$ & $1 \times 1$ & AUS & 1921 & $110+$ & . & $\odot$ & 0 \\
\hline
\end{tabular}

\begin{tabular}{|c|c|c|c|c|c|c|c|}
\hline$M x$ & $1 \times 1$ & AUS & 2011 & $\odot$ & 0.003418 & $\odot .0 \odot 4187$ & 0.003813 \\
\hline$M x$ & $1 \times 1$ & AUS & 2011 & 1 & $\odot .0 \odot \odot 259$ & 0.000345 & 0.000303 \\
\hline$M x$ & $1 \times 1$ & AUS & 2011 & 2 & $\odot .000161$ & $\odot .000166$ & 0.000163 \\
\hline$M x$ & $1 \times 1$ & AUS & 2011 & 3 & $\odot .000155$ & $\odot .0 \odot \odot 12$ & 0.000137 \\
\hline$M x$ & $1 \times 1$ & AUS & 2011 & 4 & $\odot .000114$ & $\odot .000 \odot 94$ & 0.000104 \\
\hline$M x$ & $1 \times 1$ & AUS & 2011 & 5 & ๑. 000108 & 0.000143 & 0.000126 \\
\hline$M x$ & $1 \times 1$ & AUS & 2011 & 6 & $\odot .000 \odot 81$ & 0.000111 & 0.000096 \\
\hline$M x$ & $1 \times 1$ & AUS & 2011 & 7 & $\odot .000052$ & 0.000113 & 0.000083 \\
\hline$M x$ & $1 \times 1$ & AUS & 2011 & 8 & $\odot .000135$ & $\odot .0 \odot \odot \odot 86$ & 0.00011 \\
\hline$M x$ & $1 \times 1$ & AUS & 2011 & 9 & 0.000098 & $\odot .0 \odot \odot \odot 72$ & 0.000084 \\
\hline$M x$ & $1 \times 1$ & AUS & 2011 & 10 & $\odot .000104$ & $\odot .000 \odot 92$ & $\odot .000098$ \\
\hline$M x$ & $1 \times 1$ & AUS & 2011 & 11 & $\odot . \odot \odot \odot \odot 3$ & $\odot .000056$ & 0.000043 \\
\hline$M x$ & $1 \times 1$ & AUS & 2011 & 12 & $\odot .000096$ & $\odot .0 \odot \odot \odot 91$ & $\odot .000094$ \\
\hline$M x$ & $1 \times 1$ & AUS & 2011 & 13 & $\odot .0 \odot \odot \odot 89$ & $\odot .0 \odot \odot \odot 91$ & $\odot .0 \odot \odot \odot 9$ \\
\hline$M x$ & $1 \times 1$ & AUS & 2011 & 14 & $\odot .0 \odot \odot 117$ & $\odot .000167$ & $\odot .0 \odot \odot 143$ \\
\hline$M x$ & $1 \times 1$ & AUS & 2011 & 15 & ๑. 000188 & $\odot .0 \odot \odot 24$ & $\odot .000215$ \\
\hline
\end{tabular}




\begin{tabular}{|c|c|c|c|c|c|c|c|}
\hline$M x$ & $1 \times 1$ & USA & 2013 & $\odot$ & ๑. .005371 & ๑. .006518 & ๑. .0०5958 \\
\hline$M x$ & $1 \times 1$ & USA & 2013 & 1 & 0.000377 & 0.000459 & 0.000419 \\
\hline$M x$ & $1 \times 1$ & USA & 2013 & 2 & $\odot .00 \odot 22$ & $\odot .0 \odot \odot 29$ & 0.000255 \\
\hline$M x$ & $1 \times 1$ & USA & 2013 & 3 & ๑. . $0 \odot 163$ & $\odot .0 \odot \odot 212$ & ๑ . $0 \odot \odot 188$ \\
\hline$M x$ & $1 \times 1$ & USA & 2013 & 4 & ๑. .000138 & ๑. .000182 & 0.00016 \\
\hline$M x$ & $1 \times 1$ & USA & 2013 & 5 & 0.000125 & ๑. . 000151 & ๑. . 000138 \\
\hline$M x$ & $1 \times 1$ & USA & 2013 & 6 & ๑. . 000112 & 0.00013 & ๑. . 000121 \\
\hline$M x$ & $1 \times 1$ & USA & 2013 & 7 & $9.50 E-05$ & 0.00012 & ๑. . 000108 \\
\hline$M x$ & $1 \times 1$ & USA & 2013 & 8 & $9.70 \mathrm{E}-05$ & ๑. . 000124 & ๑ . 000111 \\
\hline$M x$ & $1 \times 1$ & USA & 2013 & 9 & $9.10 \mathrm{E}-05$ & 0.000135 & 0.000113 \\
\hline$M x$ & $1 \times 1$ & USA & 2013 & 10 & $9.70 \mathrm{E}-05$ & ๑. . 000112 & ๑. . 000105 \\
\hline$M x$ & $1 \times 1$ & USA & 2013 & 11 & $9.90 E-05$ & ๑. . 000124 & ๑. . 000112 \\
\hline$M x$ & $1 \times 1$ & USA & 2013 & 12 & ๑. .000114 & 0.000153 & ๑. . 000134 \\
\hline$M x$ & $1 \times 1$ & USA & 2013 & 13 & ๑. .000143 & ๑. . 000179 & ๑. . 000161 \\
\hline$M x$ & $1 \times 1$ & USA & 2013 & 14 & ๑. .000149 & ๑. . 000234 & ๑ . 000192 \\
\hline$M x$ & $1 \times 1$ & USA & 2013 & 101 & ๑. 395261 & 0.470728 & 0.407509 \\
\hline$M x$ & $1 \times 1$ & USA & 2013 & 102 & ๑. 433161 & 0.47475 & ๑. 439277 \\
\hline$M x$ & $1 \times 1$ & USA & 2013 & 103 & 0.464843 & $\odot .527056$ & $\odot .473084$ \\
\hline$M x$ & $1 \times 1$ & USA & 2013 & 104 & 0.485677 & 0.52569 & 0.490556 \\
\hline$M x$ & $1 \times 1$ & USA & 2013 & 105 & 0.543724 & 0.628523 & $\odot .553062$ \\
\hline$M x$ & $1 \times 1$ & USA & 2013 & 106 & 0.550437 & $\odot .553458$ & $\odot .550762$ \\
\hline$M x$ & $1 \times 1$ & USA & 2013 & 107 & 0.557404 & 0.536039 & 0.555127 \\
\hline$M x$ & $1 \times 1$ & USA & 2013 & 108 & ๑. 701961 & 0.833003 & 0.714516 \\
\hline$M x$ & $1 \times 1$ & USA & 2013 & 109 & 0.583899 & ๑. 401284 & 0.565767 \\
\hline$M x$ & $1 \times 1$ & USA & 2013 & $110+$ & 0.654499 & 1.056471 & $\odot .698257$ \\
\hline
\end{tabular}

\section{Example 2. Pooling country-specific abridged (5x1) cohort life tables}

Right-click on the links corresponding to "Life Tables - Male" and "Life Tables - Female" in the lower (cohort) part of the table on the webpage http://www.mortality.org/cgi-

bin/hmd/hmd_download.php and download the files c_lt_male.zip and c_lt_female.zip. Unzipping of these two archives produces ten folders: mltcoh_1x1, mltcoh_1x5, mltcoh_1x10, mltcoh_5x1, mltcoh_5x5, mltcoh_5x10,fltcoh_1x1, fltcoh_1x5, fltcoh_1x10,fltcoh_5x1, fltcoh_5x5, fltcoh_5x10. Country files from the folders mltcoh_5x1 and fltcoh_5x1 contain abridged life tables (5-year age intervals) by single calendar years for 13 populations. Copy 26 male and female life tables into folder c:InputData1 1 , open R-Studio and after sourcing of the file Pool.HMD.Mx.files.R (see the "Installation" section for details) call the function

Pool.HMD.LT.files("c:/InputData2", "c:/OutputData2", "Cohort-LTs-5x1.txt", Separator=",").

During execution of the code, the following lines appear in the Console window: 


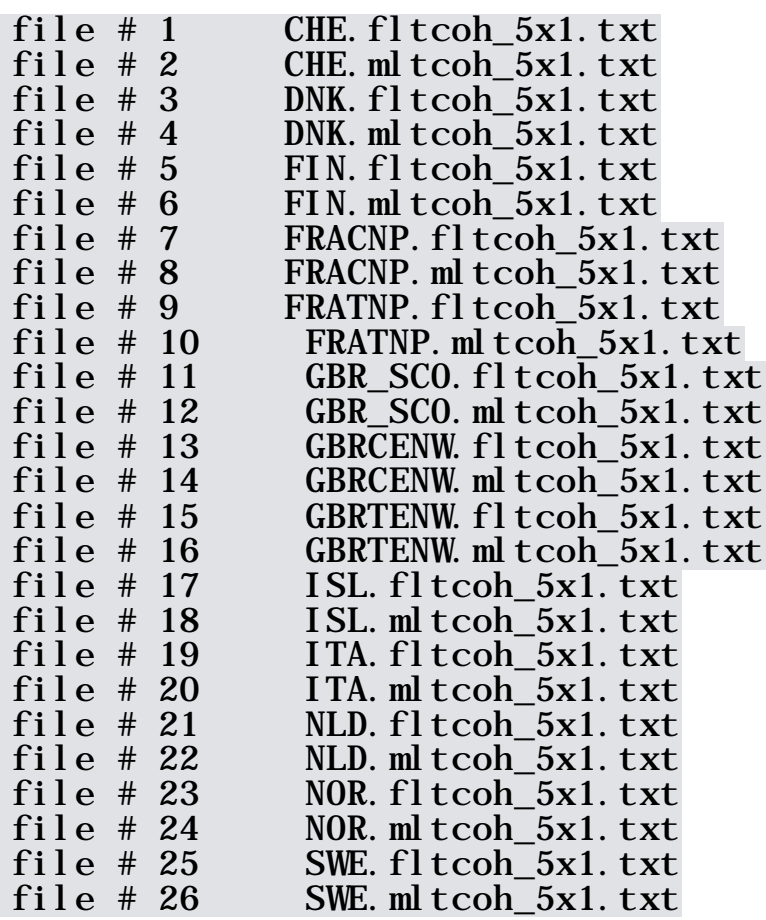

The output file Cohort-LTs-5x1.txt appears in the output folder $c: \backslash$ OutputData2. The file contains abridged life tables for males and females for all 13 populations:

$\begin{array}{rrrrrrrrrrrrrr}\text { DType } & \text { Scale } & \text { Popul } & \text { Sex } & \text { Year } & \text { Age } & \mathrm{mx} & \mathrm{qx} & \mathrm{ax} & \mathrm{Ix} & \mathrm{dx} & \text { LLx } & \text { Tx } & \text { ex } \\ \text { Itcoh } & 5 \times 1 & \mathrm{CHE} & \mathrm{f} & 1876 & 0 & 0.2306 & 0.2005 & 0.35 & 100000 & 20050 & 86967 & 4706115 & 47.06 \\ \text { Itcoh } & 5 \times 1 & \mathrm{CHE} & \mathrm{f} & 1876 & 1-4 & 0.0226 & 0.08545 & 1.4 & 79950 & 6831 & 302008 & 4619148 & 57.78 \\ \text { Itcoh } & 5 \times 1 & \mathrm{CHE} & \mathrm{f} & 1876 & 5-9 & 0.0057 & 0.028 & 2.01 & 73118 & 2047 & 359461 & 4317140 & 59.04 \\ \text { Itcoh } & 5 \times 1 & \mathrm{CHE} & \mathrm{f} & 1876 & 10-14 & 0.0034 & 0.01686 & 2.76 & 71071 & 1198 & 352668 & 3957679 & 55.69 \\ \text { Itcoh } & 5 \times 1 & \mathrm{CHE} & \mathrm{f} & 1876 & 15-19 & 0.0052 & 0.02577 & 2.62 & 69873 & 1801345074 & 3605011 & 51.59 \\ \text { Itcoh } & 5 \times 1 & \mathrm{CHE} & \mathrm{f} & 1876 & 20-24 & 0.0056 & 0.02745 & 2.52 & 68072 & 1869335728 & 3259937 & 47.89 \\ \text { Itcoh } & 5 \times 1 & \mathrm{CHE} & \mathrm{f} & 1876 & 25-29 & 0.006 & 0.0293 & 2.54 & 66203 & 1940 & 326254 & 2924209 & 44.17 \\ \text { Itcoh } & 5 \times 1 & \mathrm{CHE} & \mathrm{f} & 1876 & 30-34 & 0.0063 & 0.03077 & 2.48 & 64264 & 1977 & 316328 & 2597955 & 40.43 \\ \text { Itcoh } & 5 \times 1 & \mathrm{CHE} & \mathrm{f} & 1876 & 35-39 & 0.0061 & 0.03008 & 2.54 & 62286 & 1873 & 306830 & 2281626 & 36.63 \\ \text { Itcoh } & 5 \times 1 & \mathrm{CHE} & \mathrm{f} & 1876 & 40-44 & 0.0079 & 0.03866 & 2.52 & 60413 & 2335 & 296283 & 1974797 & 32.69 \\ \text { Itcoh } & 5 \times 1 & \mathrm{CHE} & \mathrm{f} & 1876 & 45-49 & 0.0072 & 0.03559 & 2.58 & 58077 & 2067 & 285384 & 1678514 & 28.90 \\ \text { Itcoh } & 5 \times 1 & \mathrm{CHE} & \mathrm{f} & 1876 & 50-54 & 0.0101 & 0.04933 & 2.61 & 56011 & 2763 & 273444 & 1393130 & 24.87 \\ \text { Itcoh } & 5 \times 1 & \mathrm{CHE} & \mathrm{f} & 1876 & 55-59 & 0.0141 & 0.06807 & 2.55 & 53247 & 3625 & 257374 & 1119687 & 21.03\end{array}$

$\begin{array}{llllllllllllll}\text { Itcoh } & 5 \times 1 & \text { SWE } & \mathrm{m} & 1923 & 50-54 & 0.0069 & 0.0339 & 2.68 & 82839 & 2808 & 407667 & 2328254 & 28.11 \\ \text { Itcoh } & 5 \times 1 & \text { SWE } & \mathrm{m} & 1923 & 55-59 & 0.0107 & 0.05239 & 2.66 & 80031 & 4192 & 390347 & 1920587 & 24 \\ \text { Itcoh } & 5 \times 1 & \text { SWE } & \mathrm{m} & 1923 & 60-64 & 0.0158 & 0.07594 & 2.65 & 75838 & 5759 & 365657 & 1530241 & 20.18 \\ \text { Itcoh } & 5 \times 1 & \text { SWE } & \mathrm{m} & 1923 & 65-69 & 0.0229 & 0.10857 & 2.59 & 70079 & 7609 & 332046 & 1164584 & 16.62 \\ \text { Itcoh } & 5 \times 1 & \text { SWE } & \mathrm{m} & 1923 & 70-74 & 0.034 & 0.15723 & 2.58 & 62471 & 9822 & 288571 & 832537 & 13.33 \\ \text { Itcoh } & 5 \times 1 & \text { SWE } & \mathrm{m} & 1923 & 75-79 & 0.0527 & 0.23366 & 2.57 & 52648 & 12302 & 233297 & 543966 & 10.33 \\ \text { Itcoh } & 5 \times 1 & \text { SWE } & \mathrm{m} & 1923 & 80-84 & 0.0829 & 0.34366 & 2.51 & 40347 & 13865 & 167273 & 310669 & 7.7 \\ \text { Itcoh } & 5 \times 1 & \text { SWE } & \mathrm{m} & 1923 & 85-89 & 0.1433 & 0.52449 & 2.44 & 26481 & 13889 & 96909 & 143395 & 5.41 \\ \text { Itcoh } & 5 \times 1 & \text { SWE } & \mathrm{m} & 1923 & 90-94 & 0.2404 & 0.72195 & 2.23 & 12592 & 9091 & 37811 & 46486 & 3.69 \\ \text { Itcoh } & 5 \times 1 & \text { SWE } & \mathrm{m} & 1923 & 95-99 & 0.386 & 0.87607 & 1.88 & 3501 & 3067 & 7945 & 8675 & 2.48 \\ \text { Itcoh } & 5 \times 1 & \text { SWE } & \mathrm{m} & 1923 \mathrm{~L} 00-104 & 0.5895 & 0.95959 & 1.49 & 434 & 416 & 706 & 730 & 1.68 \\ \text { Itcoh } & 5 \times 1 & \text { SWE } & \mathrm{m} & 1923 \mathrm{~L} 105-109 & 0.7467 & 1 & 1.34 & 18 & 18 & 23 & 23 & 1.34 \\ \text { Itcoh } & 5 \times 1 & \text { SWE } & \mathrm{m} & 1923 & 110+ & . & . & . & 0 & 0 & 0 & 0 & .\end{array}$

